\title{
LARVES INFESTANTES DE DIPETALONEMA SP. CHEZ DES PUCES RECOLTEES SUR DES RENARDS DU SUD-OUEST DE LA FRANCE
}

\author{
par O. Bain et J.-C. Beaucournu \\ Laboratoire de Zoologie (Vers), associé au C.N.R.S. ( $\mathrm{P}^{\mathrm{r}}$ A.-G. CHaBaUd) \\ Muséum national d'Histoire naturelle, 43, rue Cuvier, F 75231 Paris Cedex 05 \\ et Laboratoire de Parasitologie et Zoologie appliquée \\ Faculté mixte de Médecine et Pharmacie, F 35000 Rennes
}

Parmi les Puces récoltées sur des Renards du Lot-et-Garonne (à Tonneins) en avril 1972, $30 \%$ des Pulex irritans (152 Puces observées) renferment des stades infestants d'une Filaire vivipare, les autres espèces étant négatives (1 Ctenocephalides canis (Curtis, 1826) ; 3 C. f. felis (Bouché, 1835); 34 Paraceras m. melis (Walker, 1856) ; 9 Chaetopsylla trichosa Kohant, 1903 ; 53 Ch. rothschildi Kohant, 1903).

Description (fig. 1).

Corps long de 1450 à $1550 \mu$ et large de 30 à $35 \mu$; aile latérale débutant à $80 \mu$ de la tête et finissant à $65 \mu$ de l'extrémité caudale; bouche aplatie latéralement; capsule buccale haute de $8 \mu$, bien cuticularisée près de l'œsophage; anneau nerveux à $90 \mu$ de l'extrémité antérieure ; œsophages musculaire et glandulaire longs respectivement de 175 et $755 \mu$; rectum long de $26 \mu$; queue longue de $78 \mu$, terminée par une pointe arrondie; mucrons cuticulaires à la base de la pointe; 2 languettes subterminales latéro-ventrales, arrondies.

\section{Discussion.}

Les seules Filaires vivipares de Carnivores connues en Europe sont celles du Chien et peut-être du Renard (1); elles comprennent 2 Dirofilaria et 3 Dipetalonema.

1) Les 2 espèces de Dirofilaria, D. repens Railliet et Henry, 1911, et D. immitis (Leidy, 1856) ont des stades infestants qui s'opposent à nos spécimens par la très petite taille des languettes caudales et la queue courte (Nelson, 1960); en outre, le vecteur de ces Filaires est un moustiqué.

2) Le genre Dipetalonema est représenté par 3 espèces:

D. grassii (Noë, 1907) a été trouvée chez un Chien en Italie (2): la forme infestante n'est pas décrite ; le cycle s'effectue chez un Rhipicéphale (Noë, 1908).

D. dracunculoides (Cobbold, 1870) a une large distribution (Portugal, cf. Fraga de Azevedo, 1943 ; Kenya, cf. Nelson, 1963; Afrique du Nord, cf. Rioche, 1960); le stade infestant se distingue aisément de nos spécimens par la grande taille $(2350 \mu$ de long) et par la queue conique ornée de

(1) Selon Yarulin (1962), une espèce trouvée au Dagestan (U.R.S.S.), serait propre au Renard ; par la grande taille du corps, la microfilaire et la localisation dans la cavité péritonéale, elle semble proche de $D$. dracunculoides, dont elle se distinguerait par l'œsophage et la queue plus courte ; mais la description de papilles anales chez la $q$ rend le reste de l'analyse morphologique suspecte et, en l'absence de $\delta$, il n'est pas possible de confirmer la validité de cette espèce.

(2) Les Filiaires d'un Chien, au Brésil, décrites par Costa et Freitas en 1962 sous le nom de D. grassii ne semblent pas pouvoir être assimilées à l'espèce de Noë car la microfilaire est petite $(130 \mu$ au lieu de $660 \mu)$. 
languettes caudales pointues (Bain, 1972); le cycle s'effectue chez une Hippobosque (Nelson, 1963) et chez un Rhipicéphale (Bain, 1972).

D. reconditum (Grassi, 1890) semble être encore plus répandu (Europe, cf. Noë, 1907 ; Kenya, cf. Nelson, 1962; Brésil, cf. Costa et Freitas, 1962; Mexique, cf. Chabaud \& Mazzotti, 1962; U.S.A., Newton et Wright, 1957); le stade infestant, bien décrit par Calandruccio (1892) a une morphologie et des dimensions identiques à celles de nos spécimens; en outre, le cycle s'effectue chez une Puce.

\section{Conclusion.}

L'identification d'une Filaire par le stade infestant ne peut être considérée comme définitive, car de nombreuses formes infestantes sont inconnues ou insuffisamment décrites. Toutefois la grande ressemblance entre nos spécimens et ceux de D. reconditum ainsi que leur localisation chez une Puce, rend très probable l'identification à $D$. reconditum.

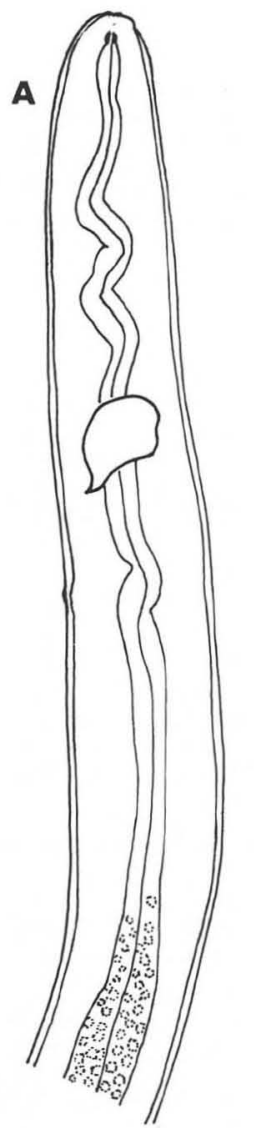

B

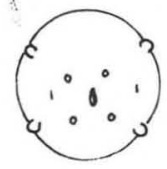

$=$
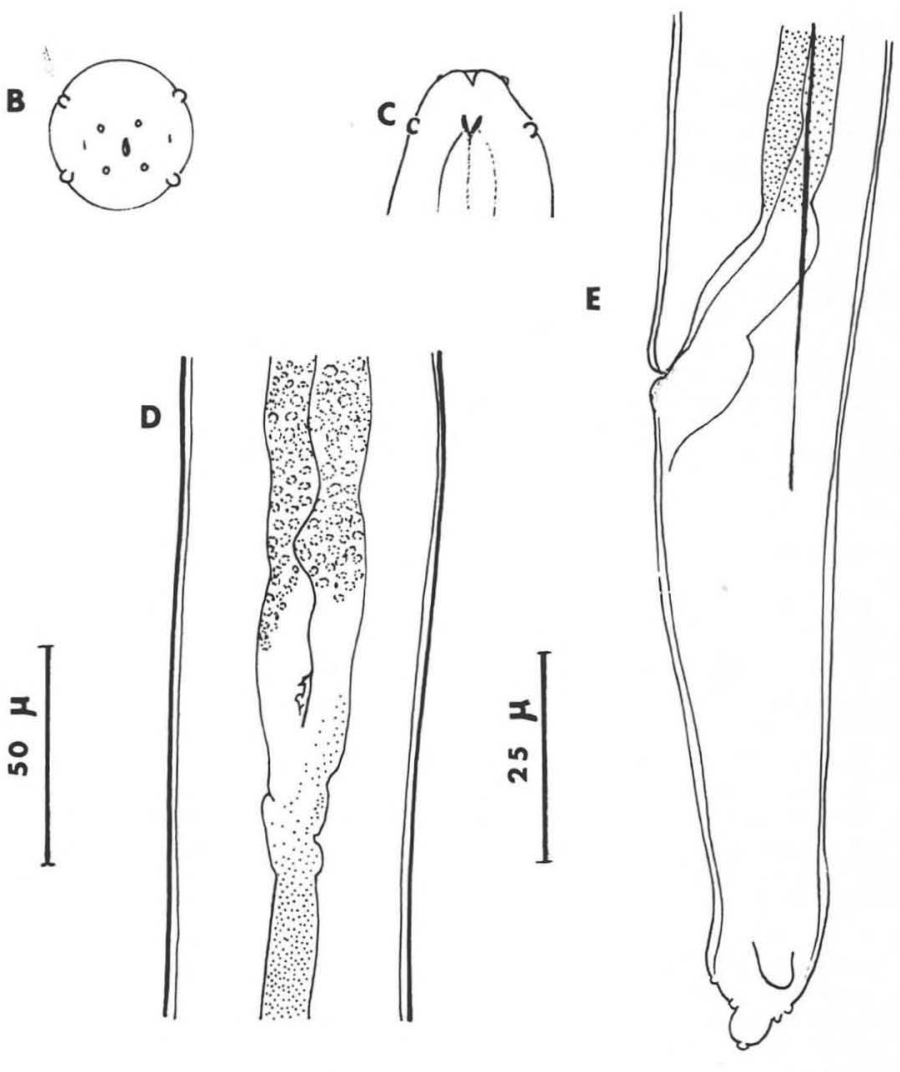

FIG. 1. - Dipetalonema sp.; larve infestante. A: région antérieure, vue latérale gauche; B: tête, vue apicale; C: idem, vue médiane; D: jonction œsophage-intestin; E : région caudale, vue latérale gauche. 
Cette identification est assez surprenante du fait que $D$. reconditum n'a pas été encore signalé en France et n'a pas encore été signalé chez le Renard mais c'est la seule identification compatible avec les données morphologiques actuelles.

\section{Bibliographie}

BaIN (O.), 1972. - Recherches sur la morphogénèse des Filaires chez l'hôte intermédiaire. Ann. Para. sit. hum. comp., 47, 251-303.

Calandruccio (S.), 1892. - Descrizione degli embrioni e delle larve della Filaria recondita (Grassi). Atti Accad. Sc. Nat. Catania, 5, 1-15.

Chabaud (A. G.) et Mazzotti (L.), 1962. - Présence de Dipetalonema reconditum chez le chien au Mexique. Ann. Parasit., 37, 673-674.

Costa (H. M. A.) et Freitas (M. G.), 1962. - Dipetalonema reconditum (Grassi, 1890) e Dipetalonema grassii (Noë, 1907) em caes de Minas Gerais (Nematoda-Filarioidea). Arq. Ex. Vet. Minas Gerais, 14, 91-101.

FragA DE Azevedo (J.), 1943. - On the presence of Dipetalonema dracunculoides (Cobbold, 1870) among dogs in Portugal; contribution to the study of its morphology. Ann. Inst. Méd. Trop. Lisbonne, 1, 105-114.

Nelson (G. S.), 1960. - The identification of filarial larvae in their vectors. Ind. J. Malariol., 14, 585-582.

-, 1962. - Dipetalonema reconditum (Grassi, 1889) from the dog with a note on its development in the flea, Ctenocephalides felis and the louse, Heterodoxus spiniger. J. Helm., 36, 297-308.

-, 1963. - Dipetalonema dracunculoides (Cobbold, 1870) from the dog in Kenya: with a note on its development in the louse-fly, Hippobosca longipennis. J. Helm., 37, 235-240.

Newton (W. L.) et Wright (W. H.), 1957. - A reevaluation of the canine filariasis problem in the United States. Vet. Med., 52, 75-78.

NoË (G.), 1907. - La Filaria grassii n. sp., e la Filaria recondita Grassi. Acc. dei Lincei, 16, 806810.

-, 1908. - Il ciclo evolutivo della Filaria grassii, mihi, 1907. R.C. Accad. Lincei, 17, 282-291.

Rioche (M.), 1960. - Présence de Dipetalonema dracunculoides (Cobbold, 1870) chez le Chien dans la région d'Alger. Arch. Inst. Pasteur Algérie, 38, 386-398.

YARULIN (G. R.), 1962. - (Acanthocheilonema dagestanica n. sp. in foxes). Uchen. Zap. dagestan Univ., 11, 15-26. 